\title{
Synthesis and Characterization of Fluorescent PVA/PVAc-Rodhamine Microspheres
}

\author{
Luisa Ambrosio ${ }^{a, b *}$, María Gisela Verón ${ }^{a}$, Nicolás Silin ${ }^{a}$, Miguel O. Prado ${ }^{a, c}$ \\ ${ }^{a}$ Consejo Nacional de Investigaciones Cientificas y Técnicas (CONICET), Av. De los Pioneros, 2300, \\ CP: 8400, Bariloche, Argentina \\ ${ }^{b}$ Universidad Nacional de Buenos Aires, Paraguay, 215, CP: C1121ABG, Ciudad Autónoma, Buenos Aires, \\ Argentina \\ ${ }^{c}$ Centro Atómico Bariloche (CNEA), Av. Bustillo, 9500, CP: R8402AGP, Bariloche, Río Negro, \\ Argentina
}

Received: February 13, 2019; Revised: May 01, 2019; Accepted: June 10, 2019

\begin{abstract}
Fluorescent polymeric microspheres are used in a wide range of biomedical and technological applications, including blood flow determination, screening, flow visualization, flow tracing, medical imaging, fluorescence microscopy and flow cytometry among others. Rhodamine 6G (R6G) is a lipophilic cationic dye, which is among the most stable organic fluorophores, and with a high quantum yield in fluorescence (absorption and emission) in the visible range $(500-600 \mathrm{~nm}$ ). It is mainly applied in laser technology, and also in fluorescent bioimage. In this work we present an efficient synthesis route to obtain Polyvinyl Alcohol/Polyvinyl Acetate (PVA/PVAc) spherical microparticles containing Rhodamine $6 \mathrm{G}$ in a concentration sufficient to exploit their fluorescent properties. The synthesis method comprises the suspension polymerization of Vinyl acetate (VAc) monomer in the presence of Rhodamine, followed by saponification. Microspheres loaded with $1.9 \% \mathrm{w} / \mathrm{w}$ of Rhodamine $6 \mathrm{G}$ were obtained. They presented good sphericity and a size distribution in the range of 20 to 1550 microns. The fluorescence intensity of the microspheres was measured by UV-Vis and fluorescence spectroscopy, and detected using an image-based experimental setup, respectively. The results obtained with these methodologies showed that our microspheres exhibit fluorescence and that they are quite detectable in water flows. However, the response of UV-Visible fluorescence and absorbance was affected by the presence of the PVA / PVAc polymer matrix.
\end{abstract}

Keywords: Polyvinyl Alcohol, Microspheres, Fluorescence, Rhodamine molecule.

\section{Introduction}

Micro and nanometric polymeric particles are useful for a broad range of applications ${ }^{1-4}$. PVA microparticles are especially convenient for therapeutic embolization, reducing blood bleeding or blocking blood feeding to tumors ${ }^{5-9}$. For these purposes, there are a series of brands that offer vials containing particles with selected sizes that are adequate to specific medical requirements ${ }^{10-12}$. Moreover, in the area of fluid engineering, tracking polymeric particles immersed in a liquid phase, allows the point to point measurement of fluid velocity.

If the particles contain a fluorescent molecule as Rhodamine, using the pumping power from a laser with 532 $\mathrm{nm}$ wavelength, they fluoresce in the region $535-640 \mathrm{~nm}$. Taking sequential photographs at small and known periods of time, such as milliseconds, their position tracking can be done and thus, the determination of their velocity ${ }^{13-14}$.

When the fluid of interest is water, for example, with a density $\approx 1 \mathrm{gcm}^{-3}$, the suspended particles should have a density near that of water, being the PVA particles a good option. ${ }^{15,16}$.
PVA particles are used in the medical field, because they are biodegradable and biocompatible, could also be tracked by their fluorescent behavior in specific experimental setups ${ }^{13}$.

This paper presents a simple way to incorporate a fluorescent molecule in the bulk of PVA microspheres, and the results from the characterization of their fluorescent response, size distribution, morphology, crystallinity and glass transition temperature.

\section{Experimental Section}

\subsection{Materials}

For the preparation of the particles we used: Benzoyl peroxide (BPO, 97\%); Polyvinyl alcohol (PVA, Mw 578 $\mathrm{kg} / \mathrm{mol}$ and degree of hydrolysis $85 \%$ ); Vinylacetate (VAc, 99\% stabilized with $17 \mathrm{ppm}$ of hydroquinone); Rhodamine 6G (analytical standard); Ethanol (ACS reagent, $\geq 99.5 \%$ ) and Sodium hydroxide ( $\mathrm{NaOH}, 99 \%)$. All the reagents were purchased from Sigma Aldrich. Distilled water was used for preparing all the solutions used in this work. 


\subsection{Preparation of $P V A / P V A c-$ Rhodamine microspheres}

\subsubsection{Suspension polymerization of VAc}

We prepared a dissolution of $0.2 \mathrm{~g}$ of PVA in $100 \mathrm{ml}$ of distilled water, inside a $0.5 \mathrm{~L}$ glass reactor heated to 343.15 $\mathrm{K}$. The reactor was equipped with a reflux condenser and nitrogen flux to maintain an inert, no oxidant, atmosphere. $25 \mathrm{ml}$ of a $1 \times 10^{-3} \mathrm{M}$ Rhodamine ethanolic solution was added to another solution containing $48 \mathrm{ml} \mathrm{VAc}$ monomer and $0.5 \mathrm{~g}$ of BPO, the latter being the initiator of the polymerization reaction. The mixture was stirred until complete homogenization and poured into the reactor. A suspension of small VAc particles appeared. Polymerization taking place inside each VAc particle. During the process, the system was kept under isothermal conditions with constant agitation at $700 \mathrm{rpm}$. PVAc-Rhodamine spherical particles were generated during the polymerization in suspension of the VAc monomer ${ }^{7}$. After some minutes, the suspension of PVAc-Rhodamine microspheres was observed at naked eye. The polymerization reaction was maintained for 240 minutes; then the temperature of the suspension was reduced to a value below $\mathrm{T}_{\mathrm{g}}$ of the poly vinyl acetate, namely to $303.15 \mathrm{~K}$, to perform the saponification procedure.

\subsubsection{Saponification of PVAc-Rhodamine microspheres}

The produced PVAc-Rhodamine microspheres were maintained in the reaction vessel in which polymerization took place. The nitrogen flux was stopped in order to add a solution of $200 \mathrm{ml}$ of $\mathrm{NaOH} 1 \mathrm{M}$ to the medium to partially transform PVAc into PVA by substitution of the acetate groups for hydroxyl groups. The system was maintained at $303.15 \mathrm{~K}$, with a constant agitation of $300 \mathrm{rpm}$. After two hours of reaction, the agitation rate was ceased and the particles suspension washed with distilled water and filtered under vacuum, to obtain the PVA/PVAc-Rhodamine microspheres ${ }^{7}$.

The synthesis parameters of each stage were chosen based on the previously reported literature for obtaining PVA-PVAc particles and our experience in preparation of PVA/PVAc microspheres ?

To compare the characteristics of the novel PVA/ PVAcRhodamine microspheres and the PVA/PVAc microspheres without dye, both types of microspheres were made under the same experimental conditions.

\subsection{Particle size distribution and morphology}

The morphological study of the microspheres was carried out using a SEM Philips 515 scanning electron microscope (SEM). Samples were covered with a thin $15 \mathrm{~nm}$ gold layer, using a Blazers Sputter Coater SCD 050. The microspheres size distribution and morphology were characterized with an optical microscope Leica DM 2500. Fluorescence microscopy with a Leica lamp housing and mercury burner was used as a first confirmation of Rhodamine molecule retention in the microspheres.

\subsection{Glass transition temperature $\left(T_{g}\right)$ and crystallinity degree}

The glass transition temperature values of PVAc/PVARhodamine and PVAc/PVA microspheres were determined by Differential Scanning Calorimetry (DSC) using a DSC Q2000 equipment (TA Instruments). In each essay, about 5 $\mathrm{mg}$ of microspheres were placed and sealed in an aluminum capsule. The sample was first cooled to $253.15 \mathrm{~K}$, stabilized for 5 minutes at this temperature and then scanned from $253.15 \mathrm{~K}$ to $423.15 \mathrm{~K}$ at $5 \mathrm{~K} / \mathrm{min}$.

The crystallinity of the microspheres was studied using a XRD Panalytical Empyrean diffractometer, employing $\mathrm{CuK} \alpha$ radiation $(\lambda=1.54 \AA)$, and $40 \mathrm{kV} / 30 \mathrm{~mA}$ beam voltage/ current. Analyses were performed by varying the angle 2 $\theta$, from 10 to $90^{\circ}$, with an angular step width of $0.013^{\circ}$.

\subsection{Molecular structure}

Fourier transform infrared (FTIR) spectra were obtained using a Perkin-Elmer Paragon 1000 FTIR spectrometer.

The PVA/PVAc and PVA/PVAc-Rhodamine samples were prepared by the $\mathrm{KBr}$ tablet technique, while the Rhodamine 6G solution was measured using an optical cuvette.

Spectra were obtained by recording 30 scans between 3500 and $450 \mathrm{~cm}^{-1}$, with a resolution of $4 \mathrm{~cm}^{-1}$.

\subsection{Qualitative fluorescent response}

A water suspension of $1.65 \mathrm{~g}$ of Rhodamine loaded microspheres, with a diameter between 20-1900 $\mu \mathrm{m}$ was placed in a transparent glass tube and illuminated laterally with a light beam from a green laser with wavelength 495-570 $\mathrm{nm}$. The fluorescent response was recorded taking photos using a camera BFLY-O3-1352C mounted on a tripod in a perpendicular direction to the illuminating beam. Images were acquired using no flash and the following settings: exposure time $2.3 \mathrm{~ms}$, gain: $-10.9 \mathrm{~dB}$ and one photo per second, in a dark room. Photos were saved under tiff format file.

We analyzed the Photos to determine the fluorescence intensity from Rhodamine, which is included in the red portion of the RGB tiff files. For doing that, we used a Phyton program that allowed the quantification of the red part of each pixel in the image, within the RGB (red, green, blue) colors. To each pixel corresponds an intensity value for each of these three primary colors. The intensity of each color is in the range 0 to 255 in decimal scale, expressed in an 8-bit binary number. This 8-bit number is part of a 24 bits' number that contains the information for RGB (. Tiff). The red color corresponds to a wavelength of 700 $\mathrm{nm}$, the green color $546 \mathrm{~nm}$ and the blue color $454 \mathrm{~nm}^{17,18}$. For each obtained fluorescence picture, an intensity frequency histogram was built, where the independent variable is the intensity in each pixel (0-255) and the ordinate expresses the number of pixels that exhibits a given intensity. 
The information from a set of five photos was averaged with a Phyton function in order to obtain each histogram.

For comparison, the same procedure was applied to two non-fluorescent suspensions in distilled water. $10 \mathrm{w} / \mathrm{v}$ $\%$ of hollow glass microspheres (diameter between 0.5-46 $\mu \mathrm{m}) ; 10 \mathrm{w} / \mathrm{v} \%$ microparticles of Titanium oxide (diameter between 2.0 to 2.1 microns).

\subsection{Microparticles rhodamine content and rhodamine particles distribution}

To estimate the content of Rhodamine $6 \mathrm{G}$ in the microparticles UV-Vis, fluorescence spectroscopy and elemental chemical analysis were used.

\subsubsection{UV-Vis measurements}

For the spectrophotometric determination we dissolved in $96 \%$ ethanol, PVA/PVAc- Rhodamine microspheres up to $10 \mathrm{w} / \mathrm{v} \%$. From the latter, we prepared two new diluted solutions in water with concentrations of $5 \%$ and $2.5 \mathrm{w} / \mathrm{v} \%$. The absorbance of each solution was measured at the Rhodamine maximum absorption wavelength. The approximate Rhodamine concentration in the microspheres was determined using a calibration curve.

The maximum absorption wavelength of Rhodamine was determined with a Lambda 45 Perkin-Elmer UV-Vis. An absorption scan spectrum between wavelengths 210 and $700 \mathrm{~nm}$ of a $10 \times 10^{-3} \mathrm{M}$ Rhodamine ethanolic solution was performed. After that, in the condition of maximum absorption, within the linearity range of the technique, the absorbance of a set of diluted Rhodamine solutions in water with concentrations of $0,1.88 \times 10^{-5} \mathrm{M}, 3.75 \times 10^{-5} \mathrm{M}$ and $7.5 \times 10^{-5} \mathrm{M}$ was measured to build a calibration curve of concentration vs absorbance, in order to find the approximate Rhodamine concentration in the microspheres.

\subsubsection{Fluorescence measurements}

A Perkin-Elmer LS45 Spectrometer was used to determine the fluorescence intensity levels, and emission spectrum after excitation of the microspheres with $530 \mathrm{~nm}$ wavelength light which corresponds to the wavelength with maximum absorption previously found. We built a calibration curve using diluted Rhodamine solutions in water $0,1.88 \times 10^{-5}$ $\mathrm{M}, 3.75 \times 10^{-5} \mathrm{M}$ and $7.5 \times 10^{-5} \mathrm{M}$. In order to measure the fluorescent response of the PVA/PVAc-Rhodamine microspheres they were first dissolved in ethanol $96 \%$. Afterwards three solutions with different concentrations were prepared: 10,5 and $2.5 \mathrm{w} / \mathrm{v} \% \mathrm{PVA} / \mathrm{PVAc}$-Rhodamine microspheres in water.

\subsubsection{Chemical analysis}

The nitrogen content of the microspheres was determined by combustion at $900^{\circ} \mathrm{C}$, using a Thermo Scientific FlashEA 1112 Nitrogen and Carbon analyzer ${ }^{19}$. These analyses were done at the Regional Center Bariloche of the National University of Comahue.

\section{Results and Discussion}

\subsection{Optical and scanning electron microscopy}

Microspheres with and without Rhodamine were obtained as explained in 2.2. Both types of microspheres were prepared in order to compare their fluorescent response.

Figures 1A and 1B show optical microscopy images corresponding to microspheres without and with Rhodamine respectively. At naked eye, microspheres with Rhodamine presented a yellow coloration, indicating the presence of the fluorescent molecule. Fluorescence microscopy images of PVA/ PVAc-Rhodamine microspheres confirmed this hypothesis (Figure 1C).

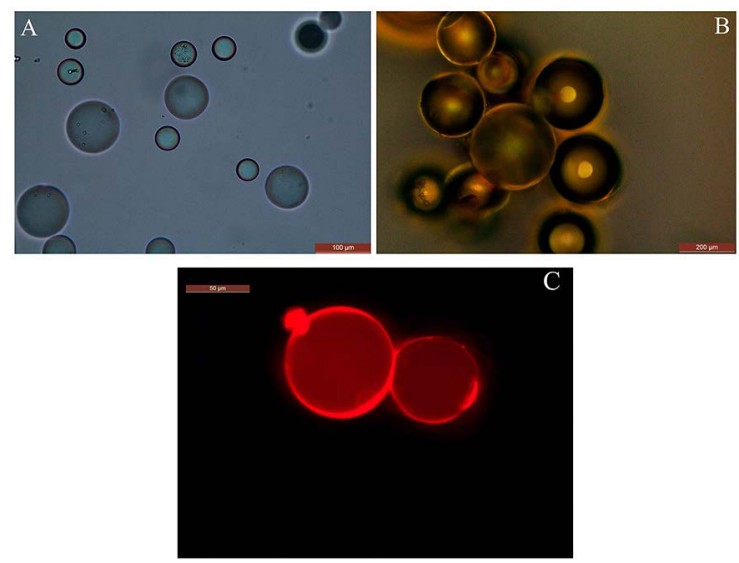

Figure 1. Optical images of A) PVA/PVAc-Rhodamine microspheres, B) PVA/ PVAc microspheres and C) Fluorescence microscopy images of PVA/PVAc-Rhodamine microspheres.

Figure 2 shows a SEM micrograph of the PVA/PVAcRhodamine (2A) and PVA/PVAc microspheres (2B) obtained after the saponification process. It can be observed that the spherical shape is maintained with the addition of Rhodamine, but particles exhibit high roughness and porosity on their surface (Figure 2A), unlike the PVA/PVAc microspheres that present a smooth surface (Figure 2B). This also suggests that the addition of the dye ethanolic solution interferes with the synthesis process, modifying the structural and morphological properties of the final material. The porosity of PVA/PVAc microspheres has been studied by other authors, it depends on the conditions and parameters of the polymerization and saponification stages ${ }^{7,20}$.

In this work, the same physical parameters were used throughout the synthesis of microspheres with and without Rhodamine. However, Rhodamine is added as an ethanolic solution, and this added alcohol could be responsible for the surface roughness and porosity of the latter particles. To evaluate this hypothesis, a sample of PVAc-Rhodamine microspheres was taken at the end of the polymerization stage.

Figure 3 shows a SEM micrograph of these PVAcRhodamine microspheres. Effectively, porosity is observed on the surface of the microspheres, which can be attributed, as reported by other authors, to the presence of the solvent ${ }^{20}$. 

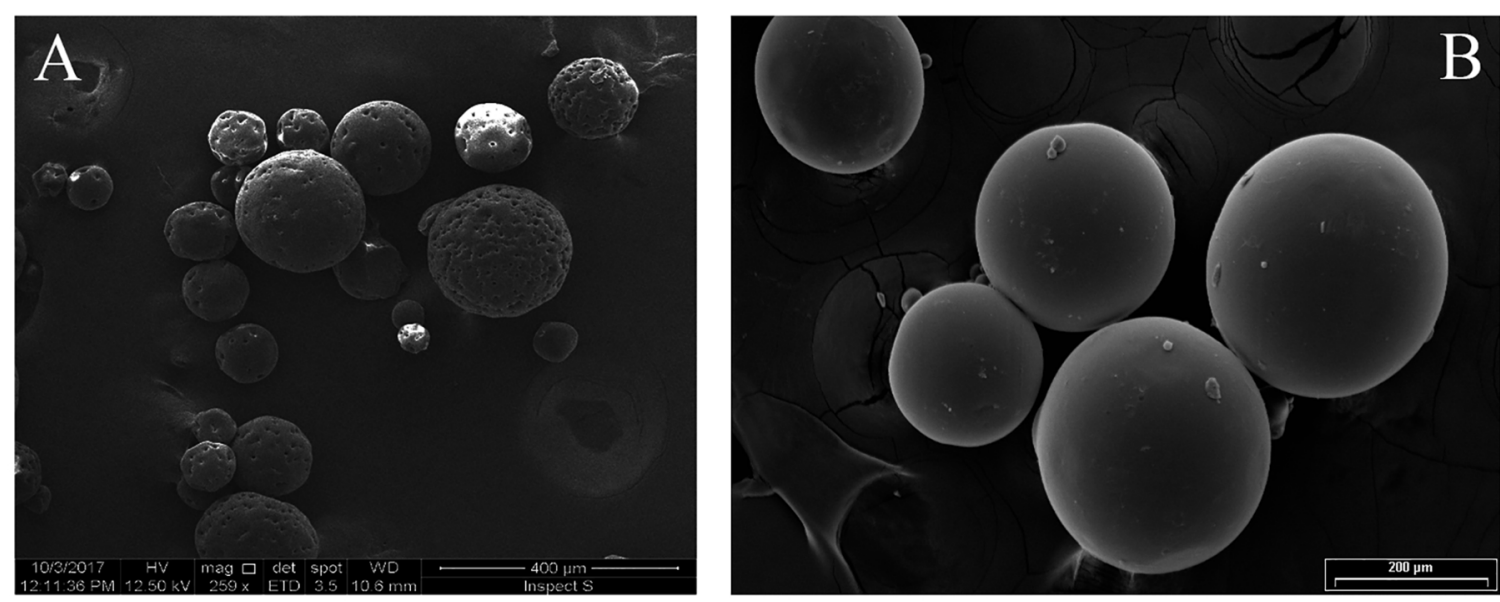

Figure 2. SEM images of A) PVA/PVAc-Rhodamine microspheres and B) PVA-PVAc microspheres.

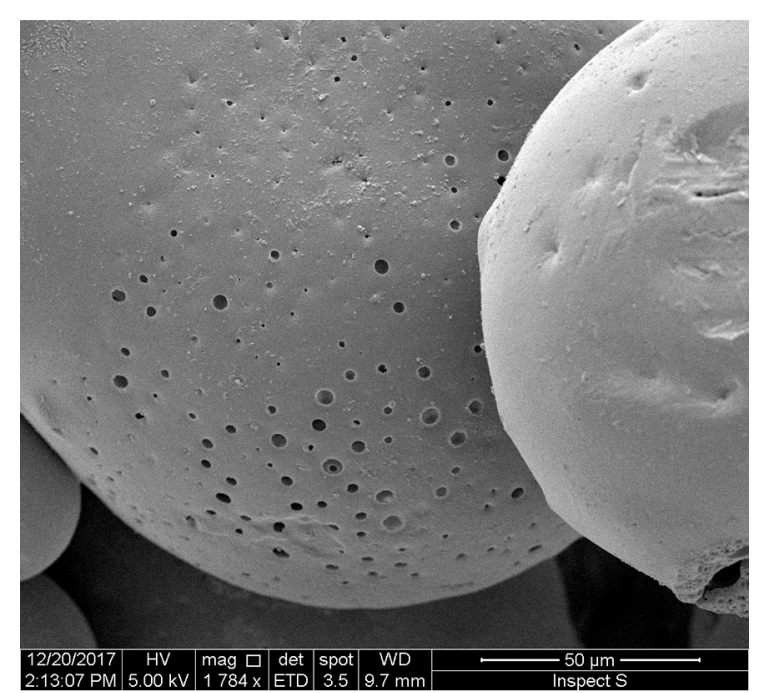

Figure 3. SEM image of PVAc -Rhodamine microspheres.

It was further found that the PVA/PVAc-Rhodamine microspheres have a bimodal, broad size distribution between 15 to 1500 microns (Figure 4B), while the microspheres without Rhodamine showed a size distribution, with sizes between 70 and 770 microns (Figure 4A). The size of the particles depends mainly on the variables of the polymerization process, namely temperature and mixing speed ${ }^{21,22,24}$. As mentioned above, the same physical parameters were used in the synthesis of both types of microspheres, so the wide size distribution of the obtained microspheres is mainly due to the addition of dye solution in the polymerization stage. It has been reported in previous work that some dyes inhibit the polymerization reaction, causing low monomer conversion rates and consequently a high degree of particle polydispersity ${ }^{23,24}$.

\subsection{XRD measurements}

X-ray Diffraction (XRD) measurements were performed to study the crystallinity of the PVAc/PVA-Rhodamine microspheres.

Figure 5 shows three XRD patterns, corresponding to PVA/PVAc-Rhodamine microspheres, PVA/PVAc microspheres (without Rhodamine), and commercial PVA.
The PVA/PVAc-Rhodamine microspheres presented a diffractogram similar to that of the PVA/PVAc microspheres, with a high Bragg diffraction intensity in the region between $2 \Theta=10-30^{\circ}$, which can be related to their amorphous structure.

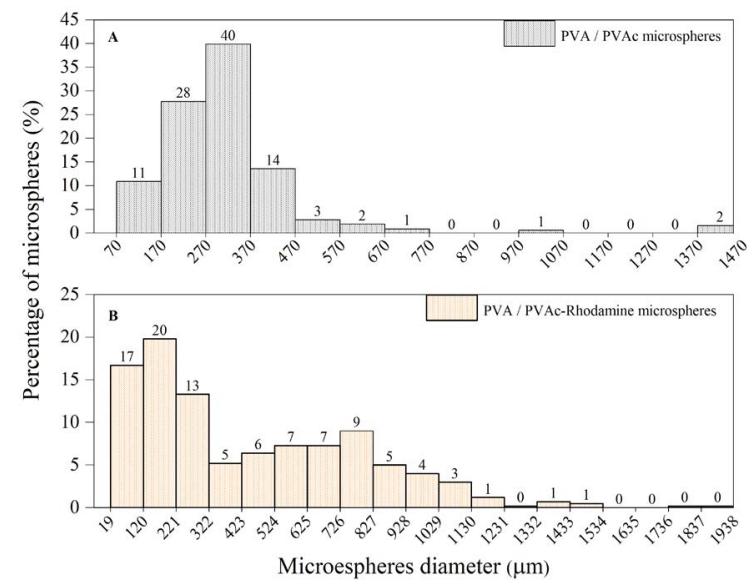

Figure 4. Size distribution of A) PVA/PVAc microspheres and B) PVA/PVAc-Rhodamine microspheres.

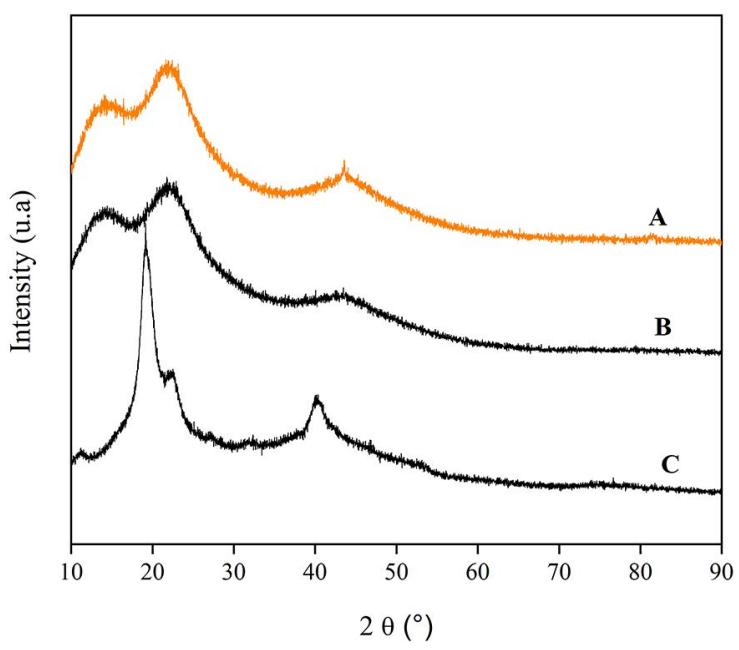

Figure 5. X-ray Diffraction of A) Commercial PVA, B) PVA/ PVAc-Rhodamine and C) PVA/PVAc microspheres. 


\subsection{Differential scanning calorimetry}

The DSC curves (Figure 6) of the PVA/PVAc-Rhodamine, and PVA/PVAc microspheres without Rhodamine, after saponification process are shown in, orange and black, respectively. It is observed that the glass transition temperature of PVA/PVAc-Rhodamine microspheres $(313.6 \mathrm{~K})$ is lower than the $\mathrm{T}_{\mathrm{g}}$ of the PVA/PVAc microspheres $(315.8 \mathrm{~K})$, which indicates the presence of dye in the matrix of polymer.

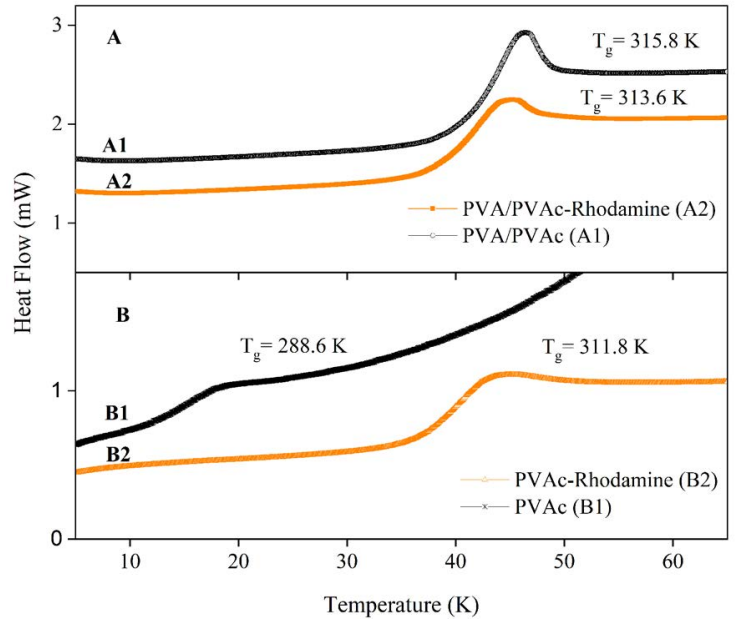

Figure 6. DSC curves of A) PVA/PVAc-Rhodamine (A2) and PVA/ PVAc microspheres (A1), B) PVAc-Rhodamine (B2) and PVAc microspheres (B1).

In addition, we observed that the $\mathrm{T}_{\mathrm{g}}$ value of non-saponified PVAc-Rhodamine samples (311.8 K) differs by $1.8 \mathrm{~K}$ from the PVA/ PVAc-Rhodamine microspheres (313.6 K), which suggests that the final material contains very little amount of hydrolyzed PVAc, that is, a very thin outer layer of PVA. On the contrary, a remarkable variation of $\mathrm{T}_{\mathrm{g}}$ was observed in the microspheres synthesized without dye, before and after the saponification stage ( $288.6 \mathrm{~K}$ and $315.8 \mathrm{~K}$ respectively), which confirms that the saponification stage is affected by the presence of Rhodamine ${ }^{25}$.

\subsection{FTIR measurements}

Figure 7 presents the spectra of PVA/PVAc, PVA/PVAcRhodamine $6 \mathrm{G}$ microspheres, and Ethanolic Rhodamine $6 \mathrm{G}$ solution used in the synthesis of the microspheres Figure 7 $\mathrm{A}, \mathrm{B}$ and $\mathrm{C}$, respectively.

In the spectrum of PVA/PVAc microspheres (Figure 7A), the characteristic vibration bands of PVAc are shown at 2927 $\mathrm{cm}^{-1}$ (stretching C-H), $1740 \mathrm{~cm}^{-1}$ (stretching $\mathrm{C}=\mathrm{O}$ ), $1434 \mathrm{~cm}^{-1}$ ( $\mathrm{CH}_{2}$ asym def), $1376 \mathrm{~cm}^{-1}$ ( $\mathrm{CH}_{3}$ asym def), $1244 \mathrm{~cm}^{-1}$ (C-H in plane bending), 1122 and $1095 \mathrm{~cm}^{-1}$ (stretching $\mathrm{C}-\mathrm{O}$ modes), $1045 \mathrm{~cm}^{-1}\left(\mathrm{CH}_{2}\right.$ wagging), $1020 \mathrm{~cm}^{-1}$ ( $\mathrm{CH}_{2}$ twisting), $946 \mathrm{~cm}^{-1}$ ( $\mathrm{CH}_{3}$ wagging), $793 \mathrm{~cm}^{-1}\left(\mathrm{CH}_{3}\right.$ rocking), 658 and $632 \mathrm{~cm}^{-1}$, in plane and out of plane $\mathrm{C}=\mathrm{O}$ bending, respectively ${ }^{26,27}$. The main bands present in the PVA, as described in the literature, are: at $3550-3200 \mathrm{~cm}^{-1}$ (stretching O-H) at $2900-2700 \mathrm{~cm}^{-1}$

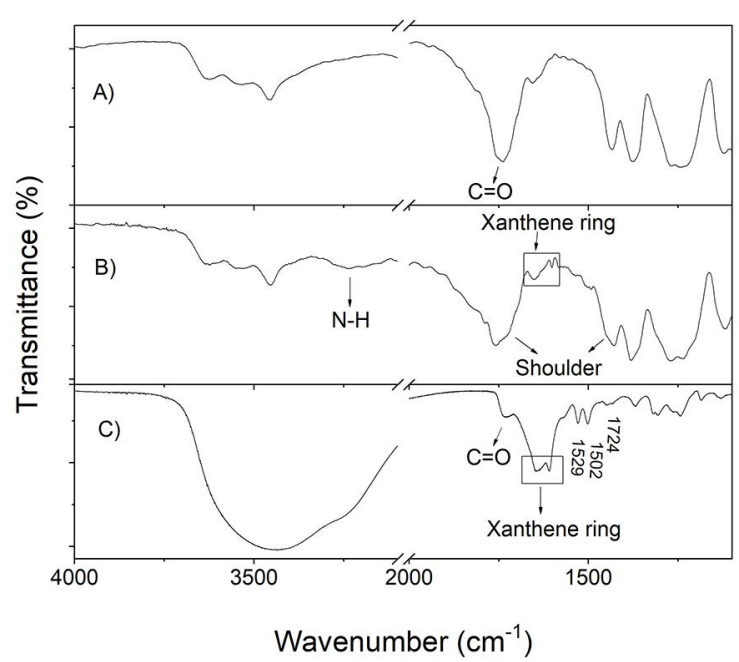

Figure 7. FTIR spectrum of PVA/PVAc-Rhodamine (red) and PVA/ PVAc microspheres (black).

(Stretching C-H), at $1650-1630 \mathrm{~cm}^{-1}$ (flexion OH), at 1461$1417 \mathrm{~cm}^{-1}$ (flexion $(\mathrm{CH})-\mathrm{CH}_{2}$ ), at $1141 \mathrm{~cm}^{-1}$ (stretching C-O) ${ }^{26}$.

In PVA/PVAc-Rhodamine microspheres, a new band is observed in the region between $3337-3115 \mathrm{~cm}^{-1}$, centered at $3232 \mathrm{~cm}^{-1}$, assigned to the N-H stretch. In the $2000-1100$ $\mathrm{cm}^{-1}$ region, a displacement of the band corresponding to the $\mathrm{C}=\mathrm{O}$ stretching from $1740 \mathrm{~cm}^{-1}$ to $1758 \mathrm{~cm}^{-1}$ is observed (Figure 7B). This shift could be due to the interaction of Rhodamine $6 \mathrm{G}$ with the carboxylic groups of PVAc. In addition, the band assigned to the $\mathrm{C}=\mathrm{O}$ stretching of $\mathrm{COOC}_{2} \mathrm{H}_{5}$ of the Rhodamine $6 \mathrm{G}$ molecule appears as a shoulder around $1724 \mathrm{~cm}^{-1}$, in accordance with the spectrum of the ethanolic solution of Rhodamine $6 \mathrm{G}$ used in the synthesis of PVA/ PVAc-Rhodamine microspheres (Figure 7C).

A new band around $1604 \mathrm{~cm}^{-1}$ was observed after the addition of Rhodamine $6 \mathrm{G}$, as well as a shift of the band positioned at $1655 \mathrm{~cm}^{-1}$ to $1650 \mathrm{~cm}^{-1}$ (Figure 7A and B). These results can be attributed to the presence of the xanthene ring ${ }^{28,29}$, and probably to the hydrogen interactions thereof with the polymer matrix. In the region between 1502 and $1580 \mathrm{~cm}^{-1}$ are included the vibrational modes of the xanthene ring coupled with ethylamine, methyl and phenyl groups (Figure 7B) ${ }^{28,29,30}$.

In addition, the band at $1450 \mathrm{~cm}^{-1}$ observed in Rhodamine $6 \mathrm{G}$ solution (Figure 7C), appears as a shoulder in the PVA/PVAcRhodamine microspheres (Figure 7B), and is assigned to the the $\mathrm{C}=\mathrm{C}$ aromatic bonds, absent in the microspheres without Rhodamine.

This analysis is in agreement with those of other authors in polymer systems doped with Rhodamine $6 \mathrm{G}^{28,29}$, and allows us to corroborate that rhodamine $6 \mathrm{G}$ is present in the polymer matrix as mentioned in section 3.3, and infer that interacts with said matrix by hydrogen bonds and dipole-dipole interactions .

\subsection{Qualitative fluorescence response}

Figure 8 shows the images obtained from three experimental setups. Two of them with no fluorescent samples: a water suspension of titanium oxide $10 \mathrm{w} / \mathrm{v} \%$ (Figure $8 \mathrm{~A}$ ), 
a water suspension of glass microspheres $10 \mathrm{w} / \mathrm{v} \%$ (Figure 8B), and one fluorescent water suspension of Rhodamine loaded microspheres (Figure 8C). The three suspensions are illuminated with a green light laser. As expected, pictures $8 \mathrm{~A}$ and $8 \mathrm{~B}$ did not show any fluorescence, instead $8 \mathrm{C}$ did show fluorescence due to the Rhodamine loaded microparticles.

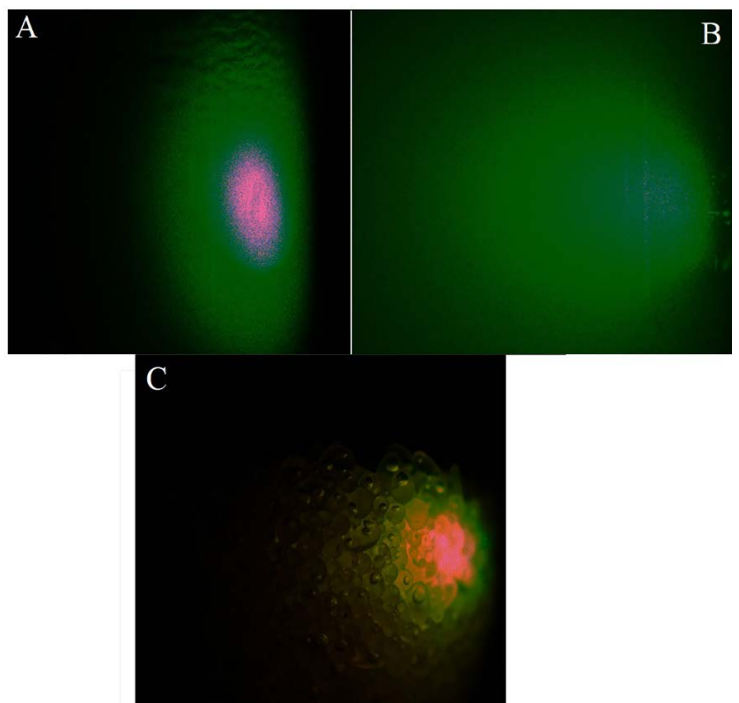

Figure 8. Fluorescence images of (A) $10 \mathrm{w} / \mathrm{v} \%$ aqueous suspension of titanium oxide particles, (B) $10 \mathrm{w} / \mathrm{v} \%$ aqueous suspension of glass microspheres and (C) $10 \mathrm{w} / \mathrm{v} \%$ aqueous suspension of PVA / PVAc- Rhodamine microspheres.

In order to equalize the fluorescence observed in each photograph, the number of pixels as a function of its intensity was plotted (Figure 9). The pixel's intensity of images $8 \mathrm{~A}$ and $8 \mathrm{~B}$ exhibit values below 50 arbitrary units, while image $8 \mathrm{C}$ exhibit peaks intensities of about 200 a.u which allowed us to confirm the fluorescent behavior of the PVA/PVAcRhodamine microspheres.

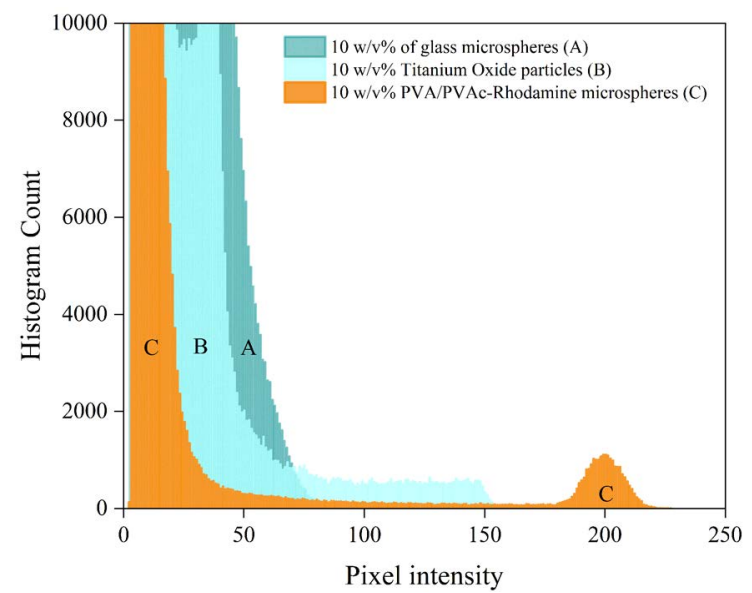

Figure 9. Histogram count vs Pixel Intensity obtained from photographs of non-fluorescent particles and PVA/PVAc-Rhodamine microspheres $(\mathrm{C})$.

\subsection{Microparticles Rhodamine content}

\subsubsection{UV-Vis absorption spectroscopy}

Figure 10 presents the UV/Vis absorption spectrum of a Rhodamine $\left(1 \times 10^{-3} \mathrm{M}\right)$ solution. Four absorption peaks were observed, three of them $(247,277$ and $348 \mathrm{~nm})$ appeared in the ultraviolet region and the last one, $530 \mathrm{~nm}$, in the visible wavelength range. The latter was used to build the calibration curve, using Rhodamine standard solutions in the range of 0 to $7.5 \times 10^{-5} \mathrm{M}$. The light absorption values were determined for each solution, and the Lambert-Beer law was fitted to this data obtaining a correlation coefficient $\mathrm{R}=0.999$. PVA/PVAcRhodamine microspheres were dissolved in a small ethanol volume, and afterwards water was added up to obtaining the final concentrations: 10,5 and $2.5 \mathrm{w} / \mathrm{v} \%$. We observed an absorbance increase with the increase of Rhodamine content in the microspheres solutions (Figure 11). However, the absorbance measured values are not proportional to the Rhodamine concentrations, thus it is not possible to determine the microspheres Rhodamine content using these results.

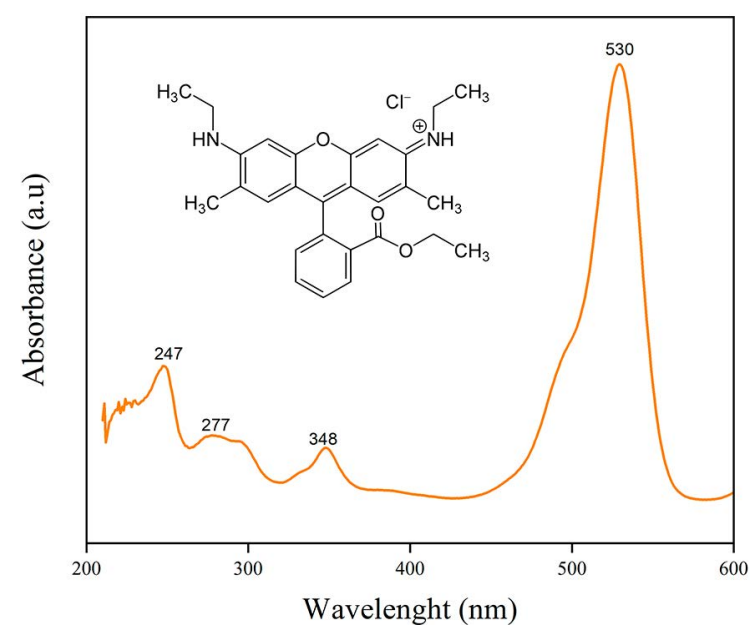

Figure 10. UV/Vis Absorption Spectrum of a $1 \times 10^{-3} \mathrm{M}$ Rhodamine solution.

\subsubsection{Fluorescence measurements}

Figure 12 presents the excitation $(534 \mathrm{~nm})$ and the fluorescent signal (553 nm) obtained from three different samples: water; a suspension of PVA/PVAc microspheres in ethanol and a suspension of PVA/PVAc -Rhodamine microspheres in ethanol. We observed that only the Rhodamine containing microspheres emit fluorescence light at $553 \mathrm{~nm}$, as expected.

Figure 13 shows the fluorescent emission spectra of two standard Rhodamine solutions $\left(1.88 \times 10^{-5} \mathrm{M}\right.$ and $3.75 \times 10^{-5}$ $\mathrm{M})$ and the corresponding to the PVA/PVAc-Rhodamine microspheres dilutions: $10,5,2.5 \mathrm{w} / \mathrm{v} \%$.

According to these results, the microspheres Rhodamine concentration is lower than $3.75 \times 10^{-5} \mathrm{M}$, which disagrees with the UV-Vis results. 


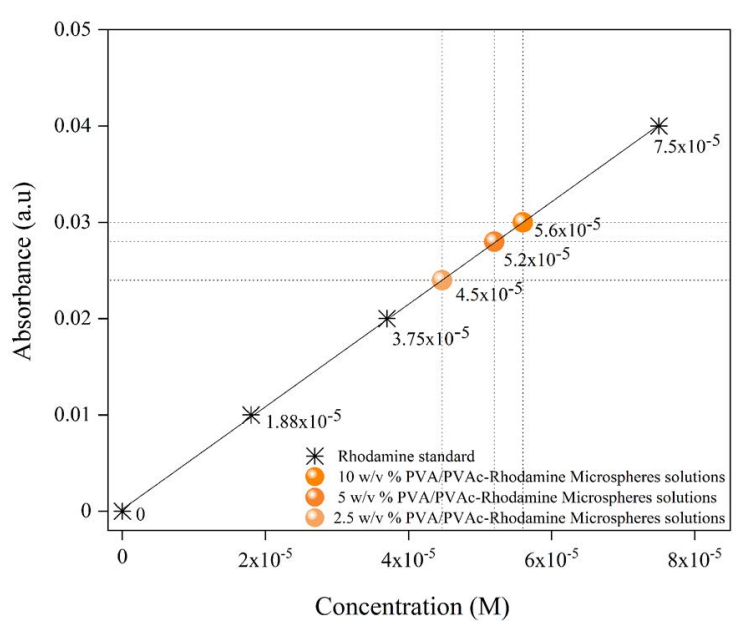

Figure 11. Calibration curve showing the measured values of absorbance corresponding to the Rhodamine 0 to $7.510^{-5} \mathrm{M}$ standard solutions. Also the absorption values measured for the $10 \mathrm{w} / \mathrm{v} \%$, $5 \mathrm{w} / \mathrm{v} \%$ and $2.5 \mathrm{w} / \mathrm{v} \%$ PVA/PVAc-Rhodamine microspheres.

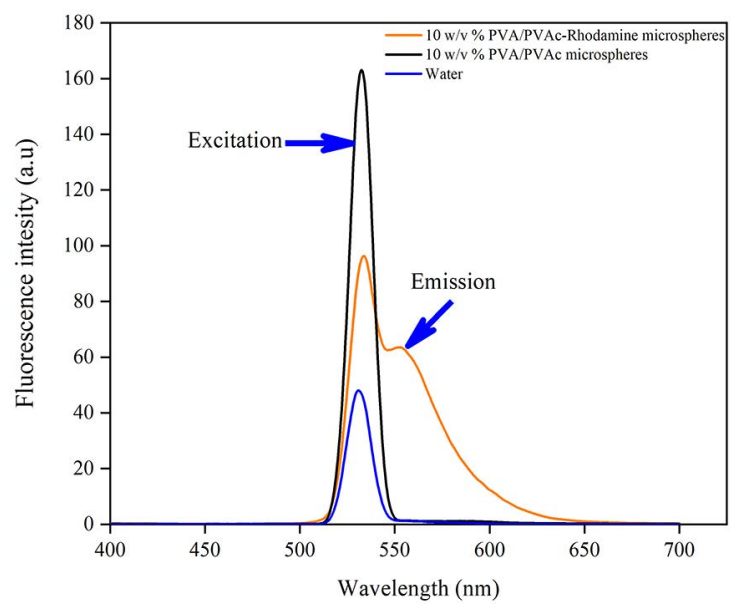

Figure 12. Excitation $(530 \mathrm{~nm})$ and Fluorescence response (553 $\mathrm{nm}$ ) of water (C), PVA/PVAc microspheres (A) and PVA/PVAc -Rhodamine microspheres dissolved in ethanol (B).

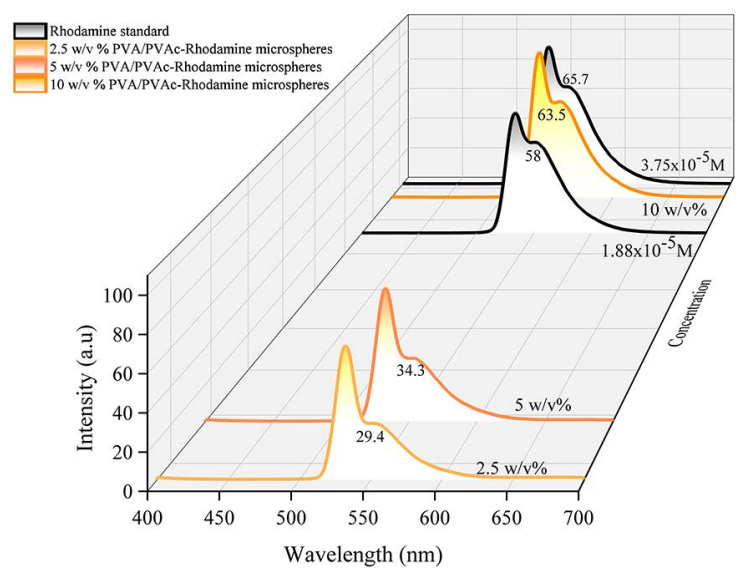

Figure 13. Fluorescence spectra of standard Rhodamine solutions $1.87 \times 10^{-5} \mathrm{M}$ and $3.75 \times 10^{-5} \mathrm{M}$ (black), and microspheres solutions $10 \mathrm{w} / \mathrm{v} \%, 5 \mathrm{w} / \mathrm{v} \%$ and $2.5 \mathrm{w} / \mathrm{v} \%$ (orange).
The lack of linearity in the last two techniques, UVVis spectrometry and fluorescence, seems to be due to the effect of PVA presence in the microspheres solutions. Thus, in order to know the actual Rhodamine concentration we used chemical analysis by combustion at $1173.2 \mathrm{~K}$ which is presented in the next section.

\subsubsection{Chemical analysis}

The elemental analysis resulted in $0.12 \pm 0.02 \mathrm{w} / \mathrm{w} \%$ total nitrogen. This value allows us to calculate the amount of Rhodamine in the microspheres, because Rhodamine was the only reagent that contained nitrogen. Thus, considering that all the nitrogen in the microspheres proceeds from Rhodamine molecules, and a Rhodamine molecular weight of 443.6 atomic mass units, this gives us a Rhodamine content in the microspheres of about $1.9 \mathrm{w} / \mathrm{w} \%$.

\section{Conclusions}

In this work, we present an efficient method to obtain fluorescent polymeric microspheres of interest in technological applications.

PVA/PVAc-Rhodamine fluorescent microspheres were prepared by suspension polymerization method followed by saponification. Using the Dumas Combustion Method, we found that the particles obtained contain approximately 1.9 $\mathrm{w} / \mathrm{w} \%$ Rhodamine $6 \mathrm{G}$. The particles show good sphericity, with a size distribution in the range of 20-1500 microns. Molecular interactions between the dye, the solvent and the polymer chain affected the polymerization and saponification steps.

The obtained $T_{g}$ values were practically the same for PVAc/Rodhamine and PVA/PVAc-Rhodamine microspheres, indicating a low saponification degree. To obtain a larger hydrolysis fraction, higher $\mathrm{NaOH}$ concentrations should be used.

The fluorescence intensity of the microspheres was measured by two analytical techniques: UV-Vis and Fluorescence spectroscopies, and by laser-induced fluorescence and an image processing tool.

The results obtained by the three methodologies showed that the synthesized microspheres exhibit fluorescence, and suggest that they are detectable in water flows.

These characteristics, together with their regular shape and size distribution, are important for their potential application in fluid dynamics studies, and even in medical applications.

\section{Acknowledgements}

This work has been partially supported by ANPCyT (National Agency of Scientific and Technological Promotion), through project PICT 2013-1403, and CNEA (National Commission of Atomic Energy). 
The authors wish to acknowledge the Materials Characterization Department of the Bariloche Atomic Center for obtaining the SEM images, and Dr. Luisa Fernández and Guillermina Urretavizcaya, for their assistance in FTIR and DSC measurements. As well to COMNIR (Center to Oncology of Nuclear Medicine of Roffo Institute of Buenos Aires) and Dra. Cristina Zarlenga and Lic. Clara Sanchez Terrero.

\section{References}

1. Saralidze K, Koole LH, Knetsch MLW. Polymeric Microspheres for Medical Applications. Materials (Basel). 2010;3(6):35373564. DOI: https://doi:10.3390/ma3063537

2. Freiberg S, Zhu XX. Polymer microspheres for controlled drug release. International Journal of Pharmaceutics. 2004;282(12):1-18. DOI: https://doi.org/10.1016/j.ijpharm.2004.04.013

3. Kawaguchi H. Functional polymer microspheres. Progress in Polymer Science. 2000;25(8):1171-1210. DOI: https://doi. org/10.1016/S0079-6700(00)00024-1

4. Ali AR, Ioppolo T, Ötügen V, Christensen M, MacFarlane D. Photonic electric field sensor based on polymeric microspheres. Journal of Polymer Science, Part B: Polymer Physics. 2014;52(3):276-279. DOI: https://doi.org/10.1002/polb.23429

5. Semenzim VL, Basso GG, da Silva DA, de Vasconcellos A, Agreli G, Lima-Oliveira APM, et al. Synthesis and characterization of novel, highly crystalline Poly (Vinyl Alcohol) microspheres for chemoembolization therapy. Journal of Applied Polymer Science. 2011;121(3):1417-1423. DOI: https://doi.org/10.1002/ app.33623

6. Lee KH, Liapi E, Buijs M, Vossen JA, Prieto Ventura V, Syed LH, et al. Percutaneous US-Guided Implantation of Vx-2 Carcinoma into Rabbit Liver: A Comparison With Open Surgical Method. Journal of Surgical Research. 2009;155(1):94-99. DOI: https:// doi.org/10.1016/j.jss.2008.08.036

7. Peixoto LS, Melo PA, Nele M, Pinto JC. Expanded Core/Shell Poly(vinyl acetate)/Poly(vinyl alcohol) Particles for Embolization. Macromolecular Materials and Engineering. 2009;294(8):463471. DOI: https://doi.org/10.1002/mame.200900028

8. Oliveira M, Cirilo LCM, Nele M, Pinto JC. Synthesis of spherical core-shell poly(vinyl acetate)/poly(vinyl alcohol) particles for use in vascular embolization: Study of morphological and molecular modifications during shell formation. Polymer Engineering and Science. 2015;55(10):2237-2244. DOI: https:// doi.org/10.1002/pen.24109

9. Meng WJ, Lu XJ, Wang H, Fan TY, Cui DC, Zhang SS, et al. Preparation and evaluation of biocompatible long-term radiopaque microspheres based on polyvinyl alcohol and lipiodol for embolization. Journal of Biomaterials Applications. 2015;30(2):133146. DOI: https://doi.org/10.1177/0885328215575622

10. Lewis AL, Adams C, Busby W, Jones SA, Wolfenden LC, Leppard $\mathrm{SW}$, et al. Comparative in vitro evaluation of microspherical embolisation agents. Journal of Materials Science: Materials in Medicine. 2006;17(12):1193-1204.
11. Lewis AL, Gonzalez MV, Lloyd AW, Hall B, Tang Y, Willis SL, et al. DC Bead: in vitro characterization of a drug-delivery device for transarterial chemoembolization. Journal of Vascular and Interventional Radiology. 2006;17(2 Pt 1):335-342. DOI: https://doi.org/10.1097/01.RVI.0000195323.46152.B3

12. Forster RE, Small SA, Tang Y, Heaysman CL, Lloyd AW, Macfarlane W, et al. Comparison of DC Bead-irinotecan and DC Bead-topotecan drug eluting beads for use in locoregional drug delivery to treat pancreatic cancer. Journal of Materials Science: Materials in Medicine. 2010;21(9):2683-2690. DOI: https://doi.org/10.1007/s10856-010-4107-4

13. Pedocchi F, Martin JE, García MH. Inexpensive fluorescent particles for large-scale experiments using particle image velocimetry. Experiments in Fluids. 2008;45(1):183-186. DOI: https:/dx.doi.org/10.1007/s00348-008-0516-2

14. Petrosky BJ, Lowe KT, Danehy PM, Wohl CJ, Tiemsin PI. Improvements in laser flare removal for particle image velocimetry using fluorescent dye-doped particles. Measurement Science and Technology. 2015;26(11):115303. DOI: https://doi. org/10.1088/0957-0233/26/11/115303

15. Kurian A, George NA, Paul B, Nampoori VPN, Vallabhan CPG. Studies on Fluorescence Efficiency and Photodegradation of Rhodamine 6G doped PMMA Using a Dual Beam Thermal Lens Technique. Laser Chemistry. 2002;20(2-4):99-110. DOI: http://dx.doi.org/10.1080/02786270215153

16. Fischer M, Georges J. Fluorescence quantum yield of rhodamine $6 \mathrm{G}$ in ethanol as a function of concentration using thermal lens spectrometry. Chemical Physics Letters. 1996;260(1-2):115118. DOI: https://doi.org/10.1016/0009-2614(96)00838-X

17. García Pamplona JA. Imagen Digital. In: García Pamplona JA. Multimedia para Principiantes. Cap. 2; 2013.

18. Pérez Vega C, Zamanillo Sainz de la Maza JM. Fundamentos de Televisión Analógica y Digital. Cap. 3. Santander: Universidad de Cantabria; 2003. p. 137.

19. Page AL, Miller RH, Keeney DR, eds. Methods of Soil Analysis. Part 2. $2^{\text {nd }}$ ed. Madison: American Society of Agronomy/Soil Science Society of America; 1982.

20. Machado F, Lima EL, Pinto JC. Uma revisão sobre os processos de polimerização em suspensão. Polímeros. 2007;17(2):166-179. DOI: http://dx.doi.org/10.1590/S0104-14282007000200016

21. Lee SG, Kim JP, Lyoo WS, Kwak JW, Noh SK, Park CS, et al. Preparation of novel syndiotactic poly(vinyl alcohol) microspheres through the low-temperature suspension copolymerization of vinyl pivalate and vinyl acetate and heterogeneous saponification. Journal of Applied Polymer Science. 2005;95(6):1539-1548. DOI: https://doi.org/10.1002/app.21296

22. Lee SG, Lyoo WS. Preparation of monodisperse poly(vinyl alcohol) microspheres by heterogeneous surface saponification and iodine complex formation. Journal of Applied Polymer Science. 2008;107(3):1701-1709. DOI: https://doi.org/10.1002/app.27194

23. Campbell AI, Bartlett P. Fluorescent Hard-Sphere Polymer Colloids for Confocal Microscopy. Journal of Colloid and Interface Science. 2002;256(2):325-330. DOI: https://doi. org/10.1006/jcis.2002.8669 
24. Horák D, Švec F, Fréchet JMJ. Preparation of colored poly(styrene-co- butyl methacrylate) micrometer size beads with narrow size distribution by dispersion polymerization in presence of dyes. Journal of Polymer Science, Part A: Polymer Chemistry. 1995;33(17):2961-2968. DOI: https://doi. org/10.1002/pola.1995.080331714

25. Sperling LH. Introduction to Physical Polymer Science. $4^{\text {th }}$ ed. Hoboken: John Wiley \& Sons; 2005.

26. Bergamasco J. Sínteses e caracterização de microesferas de poli (álcool vinílico) e sua utilização como suportes para imobilização de lipase produzida por Rhizomucor miehei e seu estudo catalítico na reação de transesterificação do óleo de soja para a produção de biodiesel via rota etílica. [Dissertation]. São José do Rio Preto: Universidade Estadual Paulista "Júlio de Mesquita Filho"; 2013.

27. Dibbern-Brunelli D, Atvars TDZ, Joekes I, Barbosa VC. Mapping phases of poly (vinyl alcohol) and poly(vinyl acetate) blends by FTIR microspectroscopy and optical fluorescence microscopy.
Journal of Applied Polymer Science. 1998;69(4):645-655. DOI: https://doi.org/10.1002/(SICI)1097-4628(19980725)69:4<645:AIDAPP3>3.0.CO;2-J

28. Faccia PA, Pardini FM, Amalvy JI. Evaluation of $\mathrm{pH}$-sensitive poly(2-hydroxyethyl methacrylate-co-2-(diisopropylamino) ethyl methacrylate) copolymers as drug delivery systems for potential applications in ophthalmic therapies/ocular delivery of drugs. eXPRESS Polymer Letters. 2015;9(6):554-566. DOI: https://doi.org/10.3144/expresspolymlett.2015.52

29. Pardini F, Faccia P, Amalvy J. Evaluation of $\mathrm{pH}$-sensitive polyurethane/2-diethylaminoethyl methacrylate hybrids potentially useful for drug delivery developments. Journal of Drug Delivery Science and Technology. 2015;30(Pt A):199208. DOI: https://doi.org/10.1016/j.jddst.2015.10.016

30. Tripathi SK, Monga A, Saini GSS. Characterization of thermally evaporated thin films of Rhodamine 6G. Smart Materials and Structures. 2009;18(12):125012. DOI: https:// doi.org/10.1088/0964-1726/18/12/125012 